\title{
Screening unit during coronavirus disease 2019 pandemic: a review of current evidence and experience of a medical center in Taiwan
}

\author{
Yung-Lun Tsai ${ }^{1} \wedge$, Tse-Hao Chen ${ }^{1} \wedge$, Wei-Yang $\mathrm{Chi}^{1}{ }^{1}$, Shih-Yi Yang ${ }^{1}$, Chen-Hao Liao ${ }^{1}$, \\ Wen-Han Chang ${ }^{1,2,3,4} \wedge$, Weide Tsai ${ }^{1,2,3} \wedge$ \\ ${ }^{1}$ Department of Emergency Medicine, Mackay Memorial Hospital, Taipei, Taiwan; ${ }^{2}$ Department of Medicine, Mackay Medical College, New \\ Taipei City, Taiwan; Hospital, Taipei, Taiwan; ${ }^{3}$ Mackay Medicine, Nursing and Management College, Taipei, Taiwan; ${ }^{4}$ Institute of Mechatronic \\ Engineering, National Taipei University of Technology, Taipei, Taiwan \\ Contributions: (I) Conception and design: WH Chang, W Tsai; (II) Administrative support: YL Tsai, WY Chi; (III) Provision of study materials or \\ patients: YL Tsai, SY Yang; (IV) Collection and assembly of data: YL Tsai, CH Liao; (V) Data analysis and interpretation: YL Tsai, TH Chen; (VI) \\ Manuscript writing: All authors; (VII) Final approval of manuscript: All authors. \\ Correspondence to: Weide Tsai. No. 92, Sec. 2, Zhongshan N. Rd., Taipei City 10449, Taiwan. Email: weidetsai@gmail.com.
}

\begin{abstract}
Severe acute respiratory syndrome coronavirus 2 (SARS-CoV-2) is an RNA virus that causes a disease named coronavirus disease 2019 (COVID-19), and it was announced as a public health emergency of international concern by the World Health Organization on January 30th, 2020. Until July 2020, there are 216 countries with reported COVID-19 cases, and over 13 million confirmed cases globally. During the pandemic of COVID-19, there are currently several different strategies among the world for screening and controlling the transmission of COVID-19, including diagnostic testing stations outside the hospital, and triage stations in hospitals and emergency departments. According to current evidence, there is not a single criteria or strategy that can fit the demand of every country or hospital. Strategy can vary largely due to different geographic locations and different pandemic progressing status of every hospital, and island countries may require a totally different approach compared with continental countries. In this article, we reviewed different strategies and approaches in reaction to the COVID-19 pandemic, and we shared our experience as a medical center in Taiwan. In conclusion, every hospital should develop their own pandemicreacting response plan, and the plan should be flexible and adjust according to the rapidly changing pandemic situation.
\end{abstract}

Keywords: Coronavirus disease 2019 (COVID-19); screening station; TOCC check point; in-hospital observation unit

Received: 14 January 2021; Accepted: 19 April 2021; Published: 10 May 2021.

doi: $10.21037 /$ ht-21-3

View this article at: http://dx.doi.org/10.21037/ht-21-3

\section{Introduction}

Severe acute respiratory syndrome coronavirus 2 (SARSCoV-2) is an RNA virus that causes a disease named coronavirus disease 2019 (COVID-19). SARS-CoV-2 originally emerged from Wuhan, China, and has been transmitting through multiple countries rapidly. It was announced as a public health emergency of international concern by the World Health Organization on January 30th, 2020. Until July 2020, there are 216 countries with

^ ORCID: Yung-Lun Tsai, 0000-0001-7082-234X; Tse-Hao Chen, 0000-0002-6370-7068; Wei-Yang Chi, 0000-0001-5295-156X; WenHan Chang, 0000-0002-2071-7258; Weide Tsai, 0000-0001-7859-2032. 
reported COVID-19 cases, and over 13 million confirmed cases globally.

During the pandemic of COVID-19, there are currently several different strategies among the world for screening and controlling the transmission of COVID-19. These strategies can be separated into two main categories: diagnostic testing stations outside the hospital, and triage stations inside the hospital area.

\section{Out-of-hospital screening station}

When discussing diagnostic testing stations outside the hospital, South Korea has the first experience of setting up low-contact testing centers at densely populated areas. According to a review article focusing on South Korea's response to the COVID-19 pandemic, the drive-through testing station was first suggested on February 23, 2020 (1). At that time, new case numbers per day were rising at high speed due to large cluster events held by church congregations. Drive-through testing is a COVID-19 screening strategy which takes place outside of hospital. It only requires the patient to drive to a specific place and open the window, and nasal swab testing was performed by medical personnel wearing personal protective equipment (PPE). Kwon et. al. described how a drive-through testing center works in an article published on fournal of Korean Medical Science (2). When a patient arrived at a drive-through center, a questionnaire was firstly filled in and several further questions were asked by medical personnel. This section preliminarily filters patients with high suspicions of COVID-19, and these patients were directly transferred to a nearby hospital for further examination. Other patients then go through a medical tent or a container for nasal swab specimen collection, and contact information was collected in case of further result noticing. The COVID-19 screening idea of drive-through center was rapidly adopted by many countries, including the US and the UK, and several articles were published regarding the experience of deploying the drive-through testing center (3-5).

However, drive-through testing center encountered some problems, including not available for patients who don't possess a car, and drive-through centers takes up too much space in dense urban environments $(1,6)$. Therefore, walk-through centers were designed and being deployed at several hospitals for rapid specimen collecting (7). Walkthrough testing center uses a swab booth with negative pressure system and High-Efficiency Particulate Air filter (HEPA) equipped. It simply requires the patients to walk in the swab booth and medical personnel perform swab specimen collecting via latex gloves fixed on the swab booth. The prototype of this "swab booth" was a design of Dr. Yu-Chi Tseng and Kai-Chen Tseng (8). It is an acrylic window barrier with two fixed gloves, which separates the indoor and outdoor environment and simultaneously allow for nasopharyngeal swab specimen collection. Being a lowcontact testing center, which is outside of hospital, walkthrough testing station allows large amounts of specimen collection and patient screening. However, these lowcontact testing centers cannot provide further diagnostic examination and patient disposition like isolation ward admission due to limited medical manpower and limited equipment.

\section{Triage station in emergency department (ED)}

Compared with out of hospital screening stations, triage stations in hospitals and EDs adopted a totally different strategy in disease control. The main difference between out of hospital screening station and triage station in hospitals is that the purpose of triage station in ED is to find out highrisk patients and protect low risk patients from COVID-19 infection via nosocomial transmission, while out of hospital screening station aim to do throat swab sampling and filter out high probability patients.

Nosocomial infection is a serious problem which is threatening not only patients but also healthcare providers working in hospitals. During a pandemic like COVID-19, triage stations in the ED act as the first line to filter out high-risk patients and possible cases. Different strategies were adopted by different hospitals around the world.

The most common adopted strategy is setting up a "fever clinic" which deals with fever patients and patients with suspected pneumonia and upper respiratory infection (URI) symptoms. The fever clinic has several different formations, including setting up medical tents outside the hospital main building, or transforming part of the ED area into the fever clinic and observation room. A hospital in western Chongqing, China, had reported their design of COVID-19 triage station (9). They set up traffic control bundles to screen every person entering the hospital, and every patient was required to fill in a questionnaire focusing on TOCC. Febrile patients or patients with TOCC related to COVID-19, were separated from non-febrile patients. Two fever clinics named "COVID-2019 fever clinic" and "general fever clinic" were set up for patients with epidemiological history and without epidemiological history respectively. 
A hospital in Zhuhai, China established the fever clinic soon after the outbreak of COVID-19 in Wuhan, China (10). Several prefab houses were constructed outside the main structure of the hospital, each prefab house plays different roles including triaging patient, first-line history taking and physical examination, lab test and imaging, patient waiting area and even an office for medical personnel. Several criteria including the "red" and "yellow" indication sign were established for risk stratification, and patients labeled as different color were sent to corresponding prefab houses for medical attention. The criteria determining whether a patient would be labeled as red or yellow is based on fever, URI or gastrointestinal (GI) symptoms, and epidemiological history including travel history, occupation and contact history.

There is another example of transforming ED into a COVID-19 triage station by constructing new infrastructure adjacent to the main building of the hospital, reported by University of California San Francisco (11). Two negativepressure medical tents named as accelerated care units (ACU), were constructed outside of the hospital main building, and a rapid triage station was set up beside the medical tents. Every patient visiting the ED was screened by symptoms such as fever, URI symptoms and myalgia. Patients with fever, URI symptoms or myalgia, were sent to the ACU for complete history taking and further examination and treatment. Besides, whenever a patient was identified as in need of immediate resuscitation or intubation, the patient was sent into the negative pressure resuscitation room directly without going to the ACU. Moreover, the swabbing station was set in one of the ACUs.

\section{Experience of MacKay Memorial Hospital}

After COVID-19 was declared as a public health emergency of international concern, measures of suspected COVID-19 patient triaging were quickly carried out.

During the pandemic of COVID-19, we applied some modification to the patient flow line to obtain better efficiency. The entrance and exit for patients are unified respectively, and an infrared thermometer was set at the entrance for screening of fever. Modular houses were constructed outside of the hospital main building and connected to the entrance of the fever observation area.

A "TOCC check point" was set at the entrance of ED, at where the infrared thermometer was set. The term TOCC referred to as the abbreviation of travel history, occupation, contact history and cluster history. A questionnaire recording the TOCC history was given to every patient coming to ED seeking medical attention. Every patient was asked if the patient has symptoms including fever and upper respiratory syndromes. If a patient fulfilled the condition of either presenting with fever when visit, positive URI syndromes, or positive TOCC, then the patient would be sent to the screening station area rather than the regular ED area.

We redesigned our fever screening station for isolation of patients with fever, upper respiratory syndromes, or highrisk TOCC history. We also renamed the fever screening station as the screening station, which consists of three areas: outdoor clinic unit, negative pressure room, and inhospital observation unit. The fever screening station was first constructed during the 2003 Taiwanese severe acute respiratory syndrome (SARS) epidemic and was proved to be efficient for preventing nosocomial infection (12).

An outdoor clinic unit consisting of medical tents and modular houses was set up for first-line history taking and physical examination. Patients sent to the screening station would be examined at the outdoor clinic unit. They would first be waiting at an open area in a medical tent, and enter the modular houses for history taking, physical exam, lab tests, and medical imaging. Only one patient was called into the modular house at one time to avoid disease transmission, and all medical personnel inside the fever clinic area were required to wear personal protection equipment (PPE) including waterproof apron, shoe cover, and N95 respirator.

In case a patient needs intravenous lines, the patient would be sent inside the in-hospital observation unit. We divided the in-hospital observation unit into negative pressure rooms, high-risk observation zone and low risk observation zone. We stratified patients who were sent into the in-hospital observation unit into three groups by COVID-19 risk. The highest risk group consists of those who had a travel history to high-risk countries and areas within 14 days with fever, or radiologic evidence of pneumonia. These patients were kept inside the negative pressure room. The high-risk observation zone is designed to keep febrile patients with URI symptoms but without radiologic evidence of pneumonia, regardless of travel history. The low-risk observation zone is considered a relatively clean area, which keeps febrile patients without travel history or URI symptoms.

\section{Discussion of different screening strategies}

Our strategy is adopted from the HAZMAT zoning during 
disaster management. According to the HAZMAT zoning, the term "hot zone" refers to areas that are surrounded by hazardous materials, while "cold zone" refers to areas that have no immediate hazardous materials (13). Similar principles were seen in managing Ebola virus outbreak in Liberia, that hospitals were separated into several adjacent areas and defined as hot zone, warm zone and cold zone (14). The negative pressure rooms can be viewed as the "hot zone", and also referred to as the dirty zone, where extreme cautious must be taken and full PPE should be used in these areas. The high-risk observation zone adjacent to the negative pressure isolation room, can be referred to as a "warm zone". Patients inside the high-risk observation zone are patients with intermediate risk of COVID-19 infection. The low-risk observation zone is the farthest from the TOCC checkpoint and is adjacent to regular nonfever ED area, referred to as "cold zone". The low-risk observation zone keeps fever patients with relatively low risk of COVID-19.

The definition of hot, warm and cold zone, or dirty and clean zone, is by relativity rather than an absolute criterion. As pandemic progressed, the capacity of the dirty zone quickly reached its limit, then the high-risk observation zone was then defined as the new dirty zone. It is worth noting that the area of the dirty zone is dynamic. As the pandemic progresses and more patients with high risk of COVID-19 arrive at $\mathrm{ED}$, the area of the dirty zone is growing in size. One principle to follow is that the dirty zone must be as far as possible from the non-fever area of ED. And patients with the highest risk or even diagnosed with COVID-19, should be kept in the negative pressure rooms.

In this article, different COVID-19 screening and triaging strategies were discussed. Drive through screening stations was first developed in South Korea, and this concept has been adopted by many countries and cities, including the US and the UK with several reports published. Although the design of the swab booth was first invented in Taiwan (8), drive-through or walk-through screening stations had not been adopted in Taiwan. The reason is mainly due to the geographic features of Taiwan and the pandemic status. The strength of out of hospital screening stations is to rapidly filter out COVID-19 cases from the society by means of mass testing. However, every confirmed case in Taiwan had undergone a complete epidemiological survey and contact history tracing conducted by Centers for Disease Control (CDC) in Taiwan. Until early November 2020, when this article was written, there was no confirmed local breakout event until Oct 29 (15), and every indigenous case was fully documented and under control. Mass testing by out of hospital screening stations would cause medical resource wasting and unnecessary loading to first-line medical personnel.

Process of visiting ED has changed over time, and all patients go through the TOCC checkpoint before entering ED. Criteria for triaging patients at the first-line triaging station also varies over time. Comparing different reports from different countries, it is most common that the triage station filters incoming patients by both fever and TOCC history. Fever has been viewed as the most important symptom during the early time of COVID-19 pandemic. However, as the pandemic progressed, afebrile patients with URI symptoms had gradually become a concern. Fever and pneumonia were viewed as a typical symptom during the early time of COVID-19 outbreak. Early research results showed that up to $98.6 \%$ of patients present with fever on admission (16). However, as more data was collected, nonfebrile COVID-19 patients quickly became a problem to deal with. According to a systematic review and metaanalysis, fever was noted in only $83.3 \%$ of COVID-19 patients (95\% CI: 78.4-87.7), and radiologic evidence of bilateral pneumonia was found in only $73.2 \%$ of patients (95\% CI: 63.4-82.1) (17). Another research also showed that an increasing number of nonfebrile patients was noted as pandemic progressed (18). These results showed that screening patients solely for fever and URI symptoms would result in misses in patients without fever. Another metaanalysis focusing on analyzing asymptomatic COVID-19 patients showed that the overall percentage of asymptomatic presentation is $15.6 \%$ (95\% CI: $10.1-23.0 \%$ ) (19). To deal with afebrile or asymptomatic COVID-19 carriers, we adopted the concept that the TOCC check point should screen patients by symptoms and TOCC history simultaneously. Any patient with positive TOCC and positive URI symptoms, requires further isolation and testing, even the patient is non-febrile when presenting to ED.

Due to the geographic feature of Taiwan, an island that is not adjacent to any country by land, every immigration and passenger could be fully traced and quarantined. Ever since the outbreak of COVID-19, Taiwan has not reported local transmission outbreaks, therefore contact history and travel history surveys can be relatively feasible. By acquiring both symptoms and TOCC history, we can preliminarily categorize patients into high risk and low risk of COVID-19. Triaging patients by both symptoms and TOCC, can highly improve efficiency and efficacy of COVID-19 risk stratification and hospital transmission 
prevention.

\section{Conclusions}

In this article, we reviewed different strategies and approaches in reaction to the COVID-19 pandemic, and we shared our experience as a medical center in Taiwan. According to current evidence, there is not a single criteria or strategy that can fit the demand of every country or hospital. Strategy can vary largely due to different geographic locations and different pandemic progressing status of every hospital, and island countries may require a totally different approach compared with continental countries. Every hospital should develop their own pandemic-reacting response plan, and the plan should be flexible and adjust according to the rapidly changing pandemic situation.

\section{Acknowledgments}

Funding: None.

\section{Footnote}

Conflicts of Interest: All authors have completed the ICMJE uniform disclosure form (available at http://dx.doi. org/10.21037/ht-21-3). WHC serves as an unpaid Editorin-Chief of Health Technology. The authors have no other conflicts of interest to declare.

Ethical Statement: The authors are accountable for all aspects of the work in ensuring that questions related to the accuracy or integrity of any part of the work are appropriately investigated and resolved.

Open Access Statement: This is an Open Access article distributed in accordance with the Creative Commons Attribution-NonCommercial-NoDerivs 4.0 International License (CC BY-NC-ND 4.0), which permits the noncommercial replication and distribution of the article with the strict proviso that no changes or edits are made and the original work is properly cited (including links to both the formal publication through the relevant DOI and the license). See: https://creativecommons.org/licenses/by-nc-nd/4.0/.

\section{References}

1. Lee D, Lee J. Testing on the move: South Korea's rapid response to the COVID-19 pandemic. Transportation Research Interdisciplinary Perspectives 2020;5:100111.

2. Kwon KT, Ko JH, Shin H, et al. Drive-Through Screening Center for COVID-19: a Safe and Efficient Screening System against Massive Community Outbreak. J Korean Med Sci 2020;35:e123.

3. Hill K, Campbell R, Mutch C, et al. Drive-through testing in COVID-19: experience from NHS Lothian. Clin Med (Lond) 2020;20:290-1.

4. Ton AN, Jethwa T, Waters K, et al. COVID-19 drive through testing: An effective strategy for conserving personal protective equipment. Am J Infect Control 2020;48:731-2.

5. Turrentine M, Ramirez M, Monga M, et al. Rapid Deployment of a Drive-Through Prenatal Care Model in Response to the Coronavirus Disease 2019 (COVID-19) Pandemic. Obstet Gynecol 2020;136:29-32.

6. Lee E, Mohd Esa NY, Wee TM, et al. Bonuses and pitfalls of a paperless drive-through screening and COVID-19: A field report. J Microbiol Immunol Infect 2021;54:85-8.

7. Kim SI, Lee JY. Walk-Through Screening Center for COVID-19: an Accessible and Efficient Screening System in a Pandemic Situation. J Korean Med Sci 2020;35:e154.

8. Gan CCR, Tseng YC, Lee KI. Acrylic window as physical barrier for Personal Protective Equipment (PPE) conservation. Am J Emerg Med 2020;38:1532-4.

9. Wu X, Zhou H, Wu X, et al. Strategies for qualified triage stations and fever clinics during the outbreak of COVID-2019 in the county hospitals of Western Chongqing. J Hosp Infect 2020;105:128-9.

10. Jiang $\mathrm{H}$, Liu JW, Ren N, et al. Emergency management in fever clinic during the outbreak of COVID-19: an experience from Zhuhai. Epidemiol Infect 2020;148:e174.

11. Noble J, Degesys NF, Kwan E, et al. Emergency department preparation for COVID-19: accelerated care units. Emerg Med J 2020;37:402-6.

12. Yen MY, Lin YE, Lee CH, et al. Taiwan's traffic control bundle and the elimination of nosocomial severe acute respiratory syndrome among healthcare workers. J Hosp Infect 2011;77:332-7.

13. Ramesh AC, Kumar S. Triage, monitoring, and treatment of mass casualty events involving chemical, biological, radiological, or nuclear agents. J Pharm Bioallied Sci 2010;2:239-47.

14. You J, Mao Q. An Improved Ward Architecture for Treatment of Patients with Ebola Virus Disease in Liberia. Am J Trop Med Hyg 2016;94:701-3.

15. Taiwan hits record 200 days with no local COVID-19 
cases. 2020. Available online: https://time.com/5905129/ taiwan-coronavirus-record/

16. Wang D, Hu B, Hu C, et al. Clinical Characteristics of 138 Hospitalized Patients With 2019 Novel Coronavirus-Infected Pneumonia in Wuhan, China. JAMA 2020;323:1061-9. Erratum in: JAMA. 2021 Mar 16;325(11):1113. doi: 10.1001/jama.2021.2336.

17. Fu L, Wang B, Yuan T, et al. Clinical characteristics of coronavirus disease 2019 (COVID-19) in China:

doi: $10.21037 / \mathrm{ht}-21-3$

Cite this article as: Tsai YL, Chen TH, Chi WY, Yang SY, Liao CH, Chang WH, Tsai W. Screening unit during coronavirus disease 2019 pandemic: a review of current evidence and experience of a medical center in Taiwan. Health Technol 2021;5:7.
A systematic review and meta-analysis. J Infect 2020;80:656-65.

18. Li Y, Jiao N, Zhu L, et al. Non-febrile COVID-19 patients were common and often became critically ill: a retrospective multicenter cohort study. Crit Care 2020;24:314.

19. He J, Guo Y, Mao R, et al. Proportion of asymptomatic coronavirus disease 2019: A systematic review and metaanalysis. J Med Virol 2021;93:820-30. 